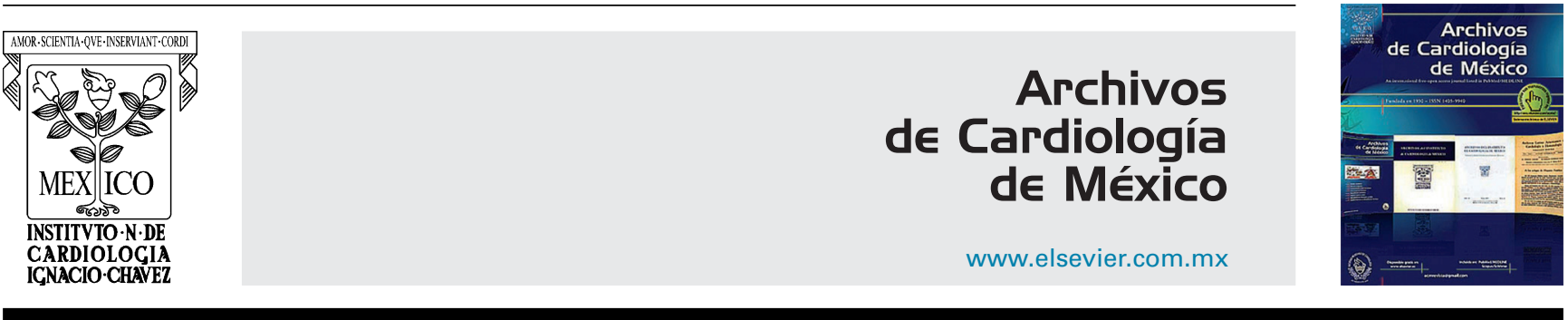

INVESTIGACIÓN CLÍNICA

\title{
Orejuela izquierda en la enfermedad reumática mitral: principal fuente embolígena en la fibrilación auricular
} CrossMark

\author{
Ovidio A. García-Villarreal* y José A. Heredia-Delgado
}

Departamento de Cirugía Cardiaca, Hospital de Cardiología UMAE 34, Instituto Mexicano del Seguro Social, , Monterrey, Nuevo León, México

Recibido el 27 de mayo de 2016; aceptado el 10 de noviembre de 2016

\section{PALABRAS CLAVE \\ Enfermedad reumática cardiaca; Fibrilación auricular; México; \\ Orejuela; \\ Trombo auricular; \\ Válvula mitral}

\begin{abstract}
Resumen
Objetivo: Demostrar que la resección quirúrgica de la orejuela izquierda en los pacientes con enfermedad reumática mitral y fibrilación auricular persistente de larga evolución disminuye la posibilidad de embolia cerebral. Asimismo, esto también elimina la necesidad de anticoagulación oral a largo plazo.

Método: Se estudiaron en forma observacional y prospectiva 27 pacientes adultos con enfermedad reumática mitral y fibrilación auricular persistente de larga evolución, sometidos a cirugía valvular mitral y resección quirúrgica de la orejuela izquierda. La anticoagulación oral con warfarina fue suspendida después del tercer mes postoperatorio, recibiendo solamente aspirina a largo plazo. El punto final fue la ausencia de embolia cerebral. Secundariamente, se evaluó la formación de trombos en la aurícula izquierda por ecocardiografía transtorácica postoperatoria. Resultados: Después del tercer mes, ningún paciente presentó embolia cerebral. Un paciente exhibió isquemia cerebral transitoria dentro de los primeros 3 meses en tratamiento con warfarina. Secundariamente, en la cirugía se encontró trombo en aurícula izquierda en 11 casos (40.7\%). De estos 11, 6 (54.5\%) habían tenido embolia cerebral previamente, sin encontrar significación estadística $(p=0.703)$.

Conclusiones: Este estudio muestra que pudiera haber indicios de que la orejuela izquierda pueda ser la principal fuente embolígena en la enfermedad reumática mitral, y que su resección pueda eliminar el riesgo de embolia cerebral en pacientes con enfermedad reumática mitral y fibrilación auricular persistente de larga evolución.

(c) 2016 Instituto Nacional de Cardiología Ignacio Chávez. Publicado por Masson Doyma México S.A. Este es un artículo Open Access bajo la licencia CC BY-NC-ND (http://creativecommons. org/licenses/by-nc-nd/4.0/).
\end{abstract}

\footnotetext{
* Autor para correspondencia. Sierra Nayarita 143, Col. Virginia Tafich; 66374, Santa Catarina, Nuevo León, México. Correo electrónico: ovidiocardiotor@gmail.com (O.A. García-Villarreal).
} 


\section{KEYWORDS}

Rheumatic heart disease;

Atrial fibrillation; Mexico;

Atrial appendage; Atrial thrombus; Mitral valve

\author{
Left atrial appendage in rheumatic mitral valve disease: The main source of \\ embolism in atrial fibrillation
}

\begin{abstract}
Objective: To demonstrate that surgical removal of the left atrial appendage in patients with rheumatic mitral valve disease and long standing persistent atrial fibrillation decreases the possibility of stroke. This also removes the need for long-term oral anticoagulation after surgery. Method: A descriptive, prospective, observational study was conducted on 27 adult patients with rheumatic mitral valve disease and long standing persistent atrial fibrillation, who had undergone mitral valve surgery and surgical removal of the left atrial appendage. Oral anticoagulation was stopped in the third month after surgery. The end-point was the absence of embolic stroke. An assessment was also made of postoperative embolism formation in the left atrium using transthoracic echocardiography.

Results: None of the patients showed embolic stroke after the third post-operative month. Only one patient exhibited transient ischaemic attack on warfarin therapy within the three postoperative months. Left atrial thrombi were also found in $11(40.7 \%)$ cases during surgery. Of these, $6(54.5 \%)$ had had embolic stroke, with no statistical significance $(P=.703)$.

Conclusions: This study suggests there might be signs that the left atrial appendage may be the main source of emboli in rheumatic mitral valve disease, and its resection could eliminate the risk of stroke in patients with rheumatic mitral valve disease and long-standing persistent atrial fibrillation.

(c) 2016 Instituto Nacional de Cardiología Ignacio Chávez. Published by Masson Doyma México S.A. This is an open access article under the CC BY-NC-ND license (http://creativecommons. org/licenses/by-nc-nd/4.0/).
\end{abstract}

\section{Introducción}

La enfermedad reumática (ER) es la forma más común de enfermedad cardiaca que afecta a la gente de estado socioeconómico bajo ${ }^{1}$. Se estima que 15.6 millones de personas en el mundo tienen ER, con aproximadamente 282,000 casos nuevos diagnosticados y 233,000 muertes por año². Quince millones de personas sufren un accidente cerebrovascular embólico cada $a \tilde{n} o^{3}$, siendo el 4.5-5.9\% relacionados con $\mathrm{ER}^{4,5}$, lo cual representa cifras de $144,000-360,000$ casos; y 108,000-269,000 muertes relacionadas, con 640,000 a 1.6 millones de sobrevivientes con algún déficit neurológico en grado variable ${ }^{2}$. La mortalidad por embolia cerebral (EC) debida a ER varía entre el $8.5 \%$ y el $47.4 \%{ }^{6-7}$. Wang et al. ${ }^{8}$ encontraron en un revisión que la sola presencia de ER parece incrementar el riesgo de EC en pacientes con fibrilación auricular (FA). De hecho, la posibilidad de EC en pacientes con ER y FA aumenta hasta 17 veces, mientras que esta posibilidad disminuye hasta 5 veces en pacientes con FA solamente ${ }^{5}$. En un estudio realizado por Maru ${ }^{9}$, la ER se encontró en el $66.3 \%$ de los casos de EC y FA. Sin embargo, el riesgo de EC en pacientes con ER sin FA no ha sido bien estudiado. La presencia de ER aumenta la posibilidad de desarrollar FA hasta en 6 veces ${ }^{10}$. La ER mitral tiene un riesgo de tromboembolismo sistémico más elevado que cualquier forma de enfermedad valvular cardiaca adquirida. La incidencia de embolismo en la estenosis mitral reumática puede variar entre el $1.5 \%$ y el $14 \%$. Con relación a los pacientes en FA, la presencia de embolia previa aumenta la posibilidad de un evento embólico nuevo en más de 3 veces $^{11}$.

El procedimiento de Cox-Maze, diseñado para eliminar cualquier tipo de flutter y FA, incluye como parte de la técnica quirúrgica la resección de la orejuela izquierda $(\mathrm{OI})^{12}$.
Además de la restauración del ritmo sinusal y de la recuperación de la función de transporte de la $\mathrm{Al}^{13}$, gran parte del éxito de procedimiento de Maze estriba en que disminuye de forma importante las tasa de EC, hecho que pudiera estar ligado a la resección de la $\mathrm{Ol}^{14,15}$. Sin embargo, dada la complejidad de esta técnica quirúrgica, existen casos de ER mitral con FA en los cuales no puede ser aplicado el procedimiento de Maze debido a factores preoperatorios que pueden aumentar la morbimortalidad operatoria (fracción de eyección del ventrículo izquierdo $<0.30$, enfermedad renal crónica, procedimiento quirúrgico múltiple, etc.) o a condiciones preoperatorias de la pared muscular de la Al (fibrosis, calcificación, tamaño excesivo) que la vuelven incapaz de recobrar funcionalidad de contracción adecuada.

Se sabe que el $91 \%$ de los trombos de la Al se forman dentro de la OI en la FA no-valvular. En principio, parecería no haber una diferencia significativa entre los pacientes con FA valvular y FA no-valvular con trombos en la Al y/u Ol con extensión a Al (13 vs. $17 \%$, respectivamente $)^{16}$. Existe una falta de evidencia en cuanto a estudios aleatorizados para determinar si la tasa de trombogenicidad de la Ol en la ER es superior, similar o inferior a la observada en la FA no-valvular. Tampoco se conoce el papel de la pared de la Al en la génesis de trombos, independientemente de la OI. En efecto, solo el $57 \%$ de los trombos en la ER se localizan en la OI, con o sin extensión a la $\mathrm{Al}^{16}$.

Este estudio analiza una serie de 27 pacientes operados por ER mitral y FA persistente de larga evolución mediante reparación valvular mitral o implante de prótesis biológica mitral asociados a la simple resección de la OI, haciendo énfasis en el manejo sin anticoagulantes orales con antivitamínicos $K$ (AVK) (warfarina) a largo plazo. Según nuestro conocimiento, este es el primer estudio en la literatura 
con una cohorte de pacientes analizando estas variables en forma simultánea.

\section{Métodos}

Se analizaron 27 casos operados con diagnóstico basal de ER mitral y FA persistente de larga evolución. A todos los casos, además de instalación de bioprótesis mitral o reparación mitral, se les realizó la resección quirúrgica de la OI. El estudio se realizó en forma observacional y prospectiva en nuestra institución, entre enero de 2014 y mayo de 2016. Todos los pacientes recibieron anticoagulación oral con AVK (warfarina) durante los primeros 3 meses postoperatorios (INR entre 2.5 y 3.5, nivel deseable 3), con monitoreo continuo del INR (primero semanalmente, hasta alcanzar nivel deseable por 3 tomas. Luego, cada mes. Si existió la necesidad de modificar la dosis, se realizó otro estudio de INR después de una semana ulterior al evento). Las características demográficas preoperatorias se muestran en la tabla 1.

El seguimiento se llevó a cabo analizando a cada paciente en visita a consulta externa y mediante ecocardiografía transtorácica (ETT) en busca de trombos en Al a los 3, 6 y 12 meses postoperatorios. Un ECG de 12 derivaciones se obtuvo en cada visita postoperatoria. El punto final primario fue analizar la tasa de eventos de EC con este tipo de cirugía. Como punto final secundario, se observó la frecuencia de trombos en la Al mediante ETT.

Tabla 1 Características preoperatorias y transoperatorias

\begin{tabular}{ll}
\hline Variable & $\mathrm{N}(\%)$ \\
\hline Edad (años $\pm D E)$ & $60.8 \pm 9.1$ \\
Género femenino & $16(59.3)$ \\
Procedimiento quirúrgico & \\
$\quad$ BPM & $19(70.4)$ \\
RVM & $8(29.6)$ \\
Resección OI & $27(100)$ \\
Trombo Al & $11(40)$ \\
Al & $2(18.2)$ \\
OI/AI & $3(27.3)$ \\
OI & $6(54.5)$ \\
ER & $27(100)$ \\
FA preoperatoria & $27(100)$ \\
AVK preoperatorios & $27(100)$ \\
ETT trombos & $7 / 11(63.6)^{\mathrm{a}}$ \\
Tamaño Al preop (cm) & $7.89 \pm 0.95$ \\
EC preoperatorio & $13(48.1)$ \\
FEVI (\% DE) & $51.6 \pm 7.6$ \\
\hline AI: auricula izquerda; AVK: antivitamincos K; BPM:
\end{tabular}

Al: aurícula izquierda; AVK: antivitamínicos K; BPM: bioprótesis mitral; DE: desviación estándar; EC: embolia cerebral; ER: enfermedad reumática; ETT: ecocardiografía transtorácica; FA: fibrilación auricular; FEVI: fracción de expulsión del ventrículo izquierdo; OI: orejuela izquierda; RVM: reparación valvular mitral.

a Relación de trombos en Ol o Al detectados por ETT/encontrados en la operación.
Las variables cuantitativas se describen como medias y desviación estándar; las variables cualitativas como frecuencias y porcentajes. Para determinar la diferencia entre grupos se utilizó la t de Student en caso de resultados cuantitativos, y Chi cuadrada para los cualitativos. Se consideró un valor de $p<0.05$ como estadísticamente significativo. Todas las pruebas se calcularon utilizando Microsoft ${ }^{\circledR}$ Excel $^{\circledR} 2011$ para Mac, versión 14.6.2.

\section{Resultados}

No hubo mortalidad operatoria. Se instaló una prótesis mitral biológica en 19 pacientes (70.4\%), y se realizó reparación de la válvula mitral en $8(29.6 \%)$. Todos los pacientes estaban en FA persistente de más de un año de evolución. Trece pacientes (48.1\%) habían presentado EC previamente a la cirugía. El tamaño preoperatorio de la Al por ETT fue de $7.89 \pm 0.95 \mathrm{~cm}$. En la cirugía, se encontró trombo en Al en 11 casos (40.7\%). De estos 11 casos, 6 (54.5\%) habían tenido EC previamente y $5(45.5 \%)$ no $(p=0.703)$. El trombo estaba localizado exclusivamente en la pared posterior de la $\mathrm{Al}$ en 2 casos (18.2\%), en la Ol con extensión a la Al en $3(27.3 \%)$, y en la Ol exclusivamente en 6 (54.5\%). Todos habían estado anticoagulados con warfarina previo a la cirugía. Todos los casos recibieron warfarina en los primeros 3 meses postoperatorios, con INR de 2.5-3.5 (nivel deseable, 3). En el seguimiento postoperatorio, 23 casos (85.2\%) estaban en FA y 4 (14.8\%) en ritmo sinusal después del tercer mes ulterior a la cirugía. Ningún caso ameritó colocación de marcapasos definitivo. Hubo solamente un caso de isquemia cerebral transitoria, dentro de los primeros 3 meses después de la cirugía, con recuperación ad integrum en las primeras $24 \mathrm{~h}$. La ETT no demostró trombosis en ningún caso a los 3 , 6 y 12 meses. No se realizó ninguna ecocardiografía transesofágica, ya que ningún paciente presentaba Ol después de la cirugía. No se llevó a cabo ningún otro estudio de gabinete neurológico, dado que solo un paciente presentó un evento transitorio, el cual fue evaluado clínicamente. En un seguimiento de 18 meses a 5 días, el $96.3 \%$ de los casos estaban libres de fenómenos tromboembólicos relacionados con la cirugía mitral. Dentro del grupo de pacientes después de los primeros 3 meses postoperatorios, sin AVK, y que están solo con un régimen de aspirina, ninguno mostró $\mathrm{EC}$ (tabla 2). Como datos adicionales, no se encontró relación directa entre el tamaño de la Al y la presencia de trombos al momento de la cirugía $(7.29 \pm 0.8 \mathrm{~cm}$ vs. $6.95 \pm 1.0 \mathrm{~cm}$, $\mathrm{p}=0.412$ ), ni entre la presencia de EC preoperatoria y el tamaño de la Al $(6.96 \pm 0.9 \mathrm{~cm}$ vs. $7.20 \pm 0.9 \mathrm{~cm}, \mathrm{p}=0.533)$ (tabla 3). Tampoco se halló relación directa entre la presencia de trombo preoperatorio en la Al y EC preoperatoria (54.5 vs. $45.5 \%, p=0.703$ ) (tabla 4 ).

\section{Discusión}

Desde su aparición en los 60s las prótesis biológicas ofrecen el atractivo de no utilizar anticoagulación oral a largo plazo, en ausencia de alguna otra razón para el uso de AVK. Según las guías para la práctica clínica, la anticoagulación oral a base de AVK está indicada en los primeros 3 meses después de la cirugía en todo caso de implante de bioprótesis mitral ${ }^{17}$. La sola presencia de FA valvular, así como la 
Tabla 2 Resultados postoperatorios

\begin{tabular}{ll}
\hline Variable & $\mathrm{N}(\%)$ \\
\hline Ritmo cardiaco postoperatorio $^{\mathrm{a}}$ & $23(85.2)$ \\
FA & $4(14.8)$ \\
$\quad$ Sinusal & \\
Terapia antitrombótica postoperatoria & \\
$\quad$ Aspirina & $26(96.3)$ \\
$\quad$ Rivaroxabán & $1(3.7)$ \\
EC & \\
Dentro de los primeros 3 meses (con AVK) & $1(3.7)$ \\
Después de los primeros 3 meses (con aspirina) & 0 \\
Trombos Al por ETT & \\
3 meses & 0 \\
6 meses & 0 \\
12 meses & 0 \\
\hline
\end{tabular}

Al: aurícula izquierda; AVK: antivitamínicos K; EC: embolia cerebral; ETT: ecocardiografía transtorácica; FA: fibrilación auricular.

a Después de los primeros 3 meses postoperatorios.

Tabla 3 Relación entre el tamaño preoperatorio de la aurícula izquierda, presencia de trombos en aurícula izquierda y embolia cerebral preoperatoria

\begin{tabular}{|c|c|c|}
\hline Variable & 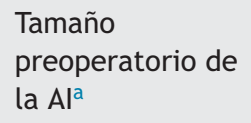 & Valor de $p$ \\
\hline Embolia cerebral & $6.96 \pm 0.9 \mathrm{~cm}$ & 0.533 \\
\hline Sin embolia cerebral & $7.20 \pm 0.9 \mathrm{~cm}$ & \\
\hline Trombos en Al & $7.29 \pm 0.8 \mathrm{~cm}$ & 0.412 \\
\hline Sin trombos en Al & $6.95 \pm 1.0 \mathrm{~cm}$ & \\
\hline
\end{tabular}

Al: aurícula izquierda.

a Valores obtenidos por ecocardiografía transtorácica por método biplanar en vista de 4 cámaras apical y 2 cámaras apical al final de la sístole, y se reporta el mayor diámetro obtenido (superoinferior).

Tabla 4 Relación entre embolia cerebral preoperatoria y la presencia de trombos en la aurícula izquierda

\begin{tabular}{lll}
\hline Variable & $\begin{array}{l}\text { Trombos en } \\
\mathrm{Al}^{*}, \mathrm{n}(\%)\end{array}$ & Valor de $\mathrm{p}$ \\
\hline EC preoperatoria & $6(54.5)$ & 0.703 \\
Sin EC preoperatoria & $5(45.5)$ & \\
\hline Al: aurícula izquierda; EC: embolia cerebral. \\
* Trombos en Al encontrados el momento de la cirugía.
\end{tabular}

ER con $\mathrm{Al}>55 \mathrm{~mm}$ por ETT son indicaciones para anticoagulación oral con AVK a largo plazo ${ }^{17}$. Aun la FA no-valvular, con $\mathrm{CHA}_{2} \mathrm{DS}_{2} \mathrm{VASC} \geq 2$ es una indicación para anticoagulación oral $^{18}$. En nuestro estudio, todos los pacientes mostraban FA preoperatoria, y el $85.2 \%$ presentó FA después de la cirugía. Más aún, el tamaño preoperatorio de la Al fue de $7.89 \pm 0.95 \mathrm{~cm}$. Teóricamente, estos pacientes deberían de ser candidatos para anticoagulación oral a largo plazo. Sin embargo, debido a la eliminación de la OI, la anticoagulación oral después de los 3 meses postoperatorios fue solamente a base de aspirina (100/mg/día).
El principal argumento de este estudio surgió a partir de la observación directa de varios pacientes que, habiendo sido sometidos a un procedimiento de Cox-Maze III e implante de bioprótesis mitral por ER mitral, en un seguimiento a largo plazo mayor a un año, habían estado libres de anticoagulación oral, y exhibían nuevamente FA sin la formación de trombos ante el escrutinio de ecocardiografía transesofágica.

A pesar de que en pacientes con ER y FA la posibilidad de EC aumenta hasta 17 veces $^{5}$, el papel de la eliminación de la Ol como generadora de trombos no ha sido bien estudiado. Existen datos indirectos a partir de los estudios del procedimiento de Maze, el cual incluye la resección de la Ol como parte de la técnica quirúrgica ${ }^{12}$. En efecto, la tasa de EC posterior al procedimiento de Maze es inferior al $0.7 \%{ }^{13-15}$. Sin embargo, no ha sido bien dilucidado si este efecto es debido a la restauración del ritmo sinusal y función de transporte de las aurículas, o a la eliminación de la OI. El presente estudio ofrece indicios de que la simple eliminación de la Ol en pacientes con ER mitral y FA persistente de larga evolución manejados sin anticoagulacioń oral a largo plazo pudiera disminuir la incidencia de EC. Hubo un caso de TIA dentro de los primeros 3 meses de la cirugía con recuperación neurológica total dentro de las primeras $24 \mathrm{~h}$. Se trató de un caso con reparación valvular mitral con Al de $82 \mathrm{~cm}$ de diámetro. En un seguimiento después de los primeros 3 meses, solamente con tratamiento a base de $100 \mathrm{mg} /$ día de aspirina, ningún caso presentó fenómenos tromboembólicos, a pesar de observar el $87.2 \%$ de los casos en FA, y con un tamaño preoperatorio de la $\mathrm{Al}>55 \mathrm{~mm}$ en el $\mathbf{8 8 . 9 \%}$ de los casos.

Se ha demostrado que en pacientes sometidos a remplazo valvular mitral por ER la presencia de FA preoperatoria estuvo asociada a una reducción importante en la supervivencia $(88.7 \%)$ comparada con aquellos en ritmo sinusal (96.6\%, $p=0.002$ ) en un seguimiento medio a 8.6 años $^{19}$. No obstante, el problema de la letalidad y efecto devastador de la FA radica en el gran número de embolias arteriales que produce. Es una enfermedad que mata en gran medida por EC. En más del $90 \%$ de los casos de FA no-valvular, la Ol es el origen de los trombos resultantes en $\mathrm{EC}^{16}$. La Ol ha sido llamada nuestro más letal apéndice humano ${ }^{20}$.

Sin embargo, sigue sin estudiarse la relación directa entre la FA valvular, la EC y la OI. Los estudios que han servido como piedra angular para el desarrollo de sistemas para la eliminación de la OI y disminución de EC por FA están enfocados exclusivamente en pacientes con FA no-valvular ${ }^{21}$.

Según un reporte de Blackshear et al. ${ }^{16}$, no parece haber una diferencia significativa entre los pacientes con FA valvular y FA no-valvular con trombos en la Al y/o Ol con extensión a la $\mathrm{Al}$ (13 vs. 17\%). En un estudio para determinar el grado de seguridad de la ecocardiografía en la detección de trombos, se determinó en forma intraoperatoria que en una serie de 474 pacientes con ER mitral el 22\% presentaba trombos en Al. De estos, el $60.9 \%$ estaba localizado en la Ol exclusivamente, el $24.7 \%$ en la Ol con extensión a Al, y el $14.2 \%$ exclusivamente en $\mathrm{Al}^{22}$. De manera similar, en el presente estudio se encontraron trombos en la $\mathrm{Al}$ en el $40.7 \%$ de los casos, de los cuales el $54.5 \%$ estaba localizado exclusivamente en la OI, el $27.3 \%$ en la Ol con extensión a Al, y el $18.2 \%$ estaba confinado a la pared posterior de la Al.

La ER mitral tiene el riesgo más elevado de tromboembolismo sistémico que cualquier forma de enfermedad valvular 
cardiaca adquirida. La incidencia de embolismo en la estenosis mitral reumática puede variar entre el $1.5 \%$ y el $14 \%{ }^{11}$. Chiang et al. ${ }^{23}$ reportan un aumento de hasta 37.1 veces la posibilidad de EC cuando existe un trombo en Al, y de 22.4 veces con la presencia de regurgitación valvular aórtica severa. En nuestro estudio se encontró que el $48.1 \%$ de nuestros pacientes había tenido una historia previa de EC. Nosotros no hallamos ninguna relación directa entre la presencia de trombo preoperatorio en la Al y la EC preoperatoria $(p=0.703)$, ni entre en tamaño de la Al y la presencia de trombos $(p=0.412)$, ni tampoco entre el tamaño de la Al y la EC preoperatoria $(p=0.533)$.

Colli et al. ${ }^{24}$ estudiaron una cohorte de 99 pacientes operados de implante de bioprótesis mitral, comparando el uso de warfarina, aspirina y sin tratamiento antitrombótico durante los primeros 90 días postoperatorios. Hubo 3 casos de EC (8.3\%) en el grupo de warfarina, uno (2\%) en el grupo de aspirina y uno (8.3\%) en el grupo sin tratamiento. En nuestro estudio solamente tuvimos un caso de isquemia cerebral transitoria en los primeros 90 días después de la cirugía, aun con tratamiento a base de warfarina.

Durães et al. ${ }^{25}$ estudiaron una cohorte de 184 pacientes operados de implante de bioprótesis mitral o aórtica. Se analizaron 16 casos (27\%) y 39 (31\%) con aspirina y sin tratamiento antitrombótico, respectivamente. Solamente hubo 3 casos (5\%) y 2 casos (1.3\%) de EC en el grupo de mitrales con aspirina y sin tratamiento antitrombótico, respectivamente, en el primer mes postoperatorio sin diferencia estadísticamente significativa. Sin embargo, en ambos estudios, solamente se reclutaron pacientes en ritmo sinusal preoperatorio ${ }^{24,25}$.

No se encontró ningún estudio en la literatura que incluya a pacientes operados de implante de bioprótesis mitral o reparación valvular mitral por ER, en FA preoperatoria, con resección quirúrgica de la $\mathrm{OI}$, sin tratamiento con AVK postoperatorio a largo plazo. Para nuestro conocimiento, este es el primer estudio que trata este rubro de pacientes con todas las variables antes mencionadas.

Una de las limitantes principales de este estudio es que no se puede considerar la comparación con otro grupo similar de pacientes dado que se tendrían que incluir pacientes con bioprótesis mitral o reparación mitral, en FA de larga evolución, con Ol presente y sin tratamiento AVK después de los primeros 3 meses de la cirugía. La sola presencia de FA, con Ol presente y permeable, es una indicación indiscutible para el uso de AVK a largo plazo ${ }^{18}$.

\section{Conclusiones}

Este es el primer estudio que muestra que pudiera haber indicios de que la Ol puede ser la principal fuente embolígena en la ER mitral, y que su resección puede eliminar el riesgo de EC en pacientes con ER mitral y FA persistente de larga evolución.

Aunque este estudio muestra indicios de que el tratamiento anticoagulante a base solamente de antiagregantes plaquetarios en este tipo de pacientes pudiera ser suficiente para evitar problemas tromboembólicos, no podemos concluir en forma contundente debido al bajo poder estadístico del mismo. Se necesita aumentar el número de pacientes y prolongar el tiempo de seguimiento de los mismos, incluso estudios multicéntricos, para poder obtener argumentos más sólidos que validen la variación en la modalidad de anticoagulación oral en estos pacientes.

\section{Responsabilidades éticas}

Protección de personas y animales. Los autores declaran que para esta investigación no se han realizado experimentos en seres humanos ni en animales.

Confidencialidad de los datos. Los autores declaran que han seguido los protocolos de su centro de trabajo sobre la publicación de datos de pacientes.

Derecho a la privacidad y consentimiento informado. Los autores declaran que en este artículo no aparecen datos de pacientes.

\section{Financiación}

Ninguna

\section{Conflicto de intereses}

Los autores declaran no tener ningún conflicto de intereses.

\section{Bibliografía}

1. WHO Expert Consultation on Rheumatic Fever and Rheumatic Heart Disease (2001: Geneva, Switzerland). Rheumatic fever and rheumatic heart disease: report of a WHO Expert Consultation, Geneva, 29 October-1 November 2001; 2001.

2. Carapetis JR, Steer AC, Mulholland EK, et al. The global burden of group A streptococcal diseases. Lancet Infect Dis. 2005;5:685-94.

3. World Health Organization The global burden of disease: 2004 update [consultado 20 May 2016]. Disponible en: http:// www.who.int/healthinfo/global_burden_disease/2004_report_ update/en/\#.

4. Wang D, Liu M, Hao Z, et al. Features of acute ischemic stroke with rheumatic heart disease in a hospitalized Chinese population. Stroke. 2012;43:2853-7.

5. Ahmad S, Wilt H. Stroke prevention in atrial fibrillation and valvular heart disease. Open Cardiovasc Med J. 2016;10:110-6.

6. Wang D, Liu M, Lin S, et al. Stroke and rheumatic heart disease: A systematic review of observational studies. Clin Neurol Neurosurg. 2013;115:1575-82.

7. Wiseman SJ, Ralston SH, Wardlaw JM. Cerebrovascular disease in rheumatic diseases. A systematic review and meta-analysis. Stroke. 2016;47:943-50.

8. Wang D, Liu M, Lin S, et al. Stroke and rheumatic heart disease: A systematic review of observational studies. Clin Neurol Neurosurg. 2013;115:1575-82.

9. Maru M. Atrial fibrillation and embolic complications. East Afr Med J. 1997;74:3-5.

10. Kannel WB, Abbott RD, Savage DD, et al. Epidemiologic features of chronic atrial fibrillation: The Framingham study. N Engl J Med. 1982;306:1018-22.

11. Gupta A, Bhatia R, Sharma G, Prasad K, Singh MB, Vibha D. Predictors of Ischemic Stroke in Rheumatic Heart Disease. J Stroke Cerebrovasc Dis. 2015;24:2810-5.

12. Cox JL. The standard maze III procedure. Oper Tech Thorac Cardiovasc Surg. 2000;5:2-22. 
13. Cox JL, Boineau JP, Schessler RE, et al. Five-year experience with the Maze procedure for atrial fibrillation. Ann Thorac Surg. 1994;56:814-24.

14. Cox JL, Ad N, Palazzo T. Impact of the maze procedure on the stroke rate in patients with atrial fibrillation. J Thorac Cardiovasc Surg. 1999;118:833-40.

15. Ad N, Cox JL. Stroke prevention as an indication for the maze procedure in the treatment of atrial fibrillation. Semin Thorac Cardiovasc Surg. 2000;12:56-62.

16. Blackshear JL, Odell JA. Appendage obliteration to reduce stroke in cardiac surgical patients with atrial fibrillation. Ann Thorac Surg. 1996;61:755-9.

17. Whitlock RP, Sun JC, Fremes SE, et al., American College of Chest Physicians. Antithrombotic and thrombolytic therapy for valvular disease: Antithrombotic Therapy and Prevention of Thrombosis, 9th ed: American College of Chest Physicians Evidence-Based Clinical Practice Guidelines. Chest. 2012;141 2 Suppl:e576S-600S.

18. January CT, Wann LS, Alpert JS, et al., ACC/AHA Task Force Members. 2014 AHA/ACC/HRS guideline for the management of patients with atrial fibrillation: executive summary: a report of the American College of Cardiology/American Heart Association Task Force on practice guidelines and the Heart Rhythm Society. Circulation. 2014;130:2071-104.
19. Wang B, Xu ZY, Han L, et al. Impact of preoperative atrial fibrillation on mortality and cardiovscular outcomes of mechanical mitral valve replacement for rheumatic mitral valve disease. Eur J Cardiothorac Surg. 2013;43:513-9.

20. Johnson WD, Ganjoo AK, Stone CD, et al. The left atrial appendage: Our most lethal human attachment! Surgical implications. Eur J Cardiothorac Surg. 2000;17:718-22.

21. Masoudi FA, Calkins H, Kavinsky CJ, et al. 2015 ACC/HRS/SCAI Left Atrial Appendage Occlusion Device Societal Overview. J Am Coll Cardiol. 2015;66:1497-513.

22. Kaymaz C, Ozdemir N, Kirma C, et al. Location, size and morphological characteristics of left atrial thrombi as assessed by echocardiography in patients with rheumatic mitral valve disease. Eur J Echocardiography. 2001;2:270-6.

23. Chiang CW, Lo SK, Ko YS, et al. Predictors of systemic embolism in patients with mitral stenosis. A prospective study. Ann Intern Med. 1998;128:885-9.

24. Colli A, D'Amico R, Mestres CA, et al. Is early antithrombotic therapy necessary after tissue mitral valve replacement? J Heart Valve Dis. 2010;19:405-11.

25. Durães AR, Durães MA, Correia LC, et al. Impact of aspirin use in the incidence of thromboembolic events after bioprosthesis replacement in patients with rheumatic disease. Rev Bras Cir Cardiovasc. 2013;28:347-52. 